\title{
SEMILATTICE OF BISIMPLE REGULAR SEMIGROUPS
}

\author{
H. R. KRISHNA IYENGAR
}

ABstract. The main purpose of this paper is to show that a regular semigroup $S$ is a semilattice of bisimple semigroups if and only if it is a band of bisimple semigroups and that this holds if and only if $D$ is a congruence on $S$. It is also shown that a quasiregular semigroup $S$ which is a rectangular band of bisimple semigroups is itself bisimple.

In $[3$, Theorem 4.4] it was shown that a semigroup $S$ is a semilattice of simple semigroups if and only if it is a union of simple semigroups. The purpose of this paper is to obtain corresponding results for a semigroup which is a semilattice of bisimple regular semigroups. Unfortunately, a semilattice of bisimple semigroups need not be a union of bisimple semigroups as illustrated by a simple $\omega$-semigroup constructed by Munn [5]. However, we get some equivalent conditions for such semigroups. In particular we show that a regular semigroup is a semilattice of bisimple semigroups if and only if it is a band of bisimple semigroups.

1. Equivalent conditions. In this section we consider a set of equivalent conditions for a semigroup $S$ to be a semilattice of bisimple semigroups. We adopt the terminology and notation of [2].

Lемма 1.1. Let $S$ be a semilattice $\Omega$ of semigroups $S_{\alpha}$ and let $D$ be a D-class of $S$. Then, either $S_{\alpha} \cap D=\square$ or $D \subseteq S_{\alpha}$.

Proof. Suppose $S_{\alpha} \cap D \neq \square$. Let $a_{\alpha} \in S_{\alpha} \cap D$. If $b_{\beta} \in S_{\beta}$ and $a_{\alpha} D b_{\beta}$, then there exists $c_{\gamma} \in S_{\gamma}(\gamma \in \Omega)$ such that $a_{\alpha} R c_{\gamma}$ and $c_{\gamma} \mathfrak{L} b_{\beta}$. Also $a_{\alpha} R c_{\gamma}$ implies that either $a_{\alpha}=c_{\gamma}$ in which case $\gamma=\alpha$, or there exist $x_{\lambda} \in S_{\lambda}, y_{\mu} \in S_{\mu}(\lambda, \mu$ in $\Omega)$ such that $a_{\alpha} x_{\lambda}=c_{\gamma}$ and $c_{\gamma} y_{\mu}=a_{\alpha}$. However, since $S$ is a semilattice of the semigroups $S_{\alpha}, a_{\alpha} x_{\lambda} \in S_{\alpha \lambda}$ and $c_{\gamma} y_{\mu}$ $\in S_{\gamma \mu}$. It follows that $\gamma=\alpha \lambda$ and $\alpha=\gamma \mu$ and so $\gamma \leqq \alpha$ and $\alpha \leqq \gamma$. Thus, in either case, $\gamma=\alpha$. Likewise, $\gamma=\beta$. Therefore, $\alpha=\beta$ and $D \subseteq S_{\alpha}$.

LEммA 1.2. Let $S$ be a semigroup. If $a b D b a$ for all $a, b$ in $S$, then $D$ is a congruence on $S$.

Proof. Let $a \mathfrak{D} b$. Then there exists $x \in S$ such that $a \mathfrak{L} x$ and $x \Re b$. Since $\mathscr{L}$ is a right congruence and $R$ is a left congruence, we have

Received by the editors July 3, 1970 .

AMS 1969 subject classifications. Primary 2092, 2093.

Key words and phrases. Quasiregular, rectangular band, semilattice, Baer-Levi semigroup, congruence. 
for $c \in S, a c \& x c$ and $c x R c b$. That is, $a c D x c$ and $c x D c b$. Since by hypothesis $x c D c x$, and $c b D b c$, then $a c D b c$ and also $c a D c b$. Thus $D$ is a congruence on $S$.

Lemma 1.3. Let $S$ be a semigroup which is a semilattice of bisimple semigroups. Then $D$ is a congruence on $S$.

Proof. Let $S=\cup_{\alpha \in \Omega} S_{\alpha}$ be a semilattice of the bisimple semigroups $S_{\alpha}$. Then, by Lemma 1.1 each $S_{\alpha}$ is a union of D-classes of $S$. However, since $S_{\alpha}$ is bisimple, any two elements of $S_{\alpha}$ are D-related in $S_{\alpha}$ and hence in $S$. Consequently, $S_{\alpha}$ consists of a single D-class of $S$. Thus the D-classes of $S$ are just the subsemigroups $S_{\alpha}$ and hence D is a congruence on $S$.

As defined in [4], an element $x$ in a semigroup $S$ is said to be quasiregular if there exist elements $a, b, c, d \in S^{1}$ such that $x=x a x b$ $=c x d x$. A semigroup $S$ is quasiregular if every element of $S$ is quasiregular. From the results in [4], we find that a regular semigroup is quasiregular, but the converse does not necessarily hold.

THEOREM 1.4. A quasiregular semigroup $S$, which is a rectangular band of bisimple semigroups, is itself bisimple.

Proof. Let $S=\bigcup\left\{S_{i \lambda} \mid i \in I, \lambda \in \Lambda\right\}$ be a rectangular band of the bisimple semigroups $S_{i \lambda}$ and let $S$ be quasiregular. Let $x_{i \lambda} \in S_{i \lambda}$, $y_{j \mu} \in S_{j \mu}$. Since $x_{i \lambda} y_{j \mu}$ is quasiregular, there exist elements $a, b, c, d$ in $S^{1}$ such that

$$
x_{i \lambda} y_{j \mu} a x_{i \lambda} y_{j \mu} b=x_{i \lambda} y_{j \mu}
$$

and

$$
c x_{i \wedge} y_{j \mu} d x_{i \lambda} y_{j \mu}=x_{i \lambda} y_{j \mu} .
$$

However, (1.4.1) implies that $x_{i \lambda} y_{j \mu} a x_{i \lambda} R x_{i \lambda} y_{j \mu}$ and (1.4.2) implies that $x_{i \lambda} y_{j \mu} \& y_{j \mu} d x_{i \lambda} y_{j \mu}$, and hence $x_{i \lambda} y_{j \mu} a x_{i \lambda} D y_{j \mu} d x_{i \lambda} y_{j \mu}$. Since $x_{i \lambda} y_{j \mu} a x_{i \lambda} \in S_{i \lambda}$ and $y_{j \mu} d x_{i \lambda} y_{j \mu} \in S_{j \mu}$, and $S_{i \lambda}$ and $S_{j \mu}$ are bisimple, it follows that $S_{i \lambda}$ and $S_{j \mu}$ are contained in a single D-class of $S$ for all $i, j \in I$ and $\lambda, \mu \in \Lambda$. Thus $S$ is bisimple.

THEOREM 1.5. The following are equivalent for any regular semigroup $S$.

(A) $S$ is a semilattice of bisimple semigroups.

(B) For $a, b \in S$, $a b D b a$.

(C) $D$ is a congruence on $S$.

(D) If $e, f, g \in E(S)$, where $E(S)$ is the set of idempotents of $S$, then eDf $\Rightarrow$ (i) eg Dfg and (ii) geDgf.

(E) $S$ is a band of bisimple semigroups. 
Proof. Assume (A) and let $S$ be a semilattice $\Omega$ of bisimple semigroups $S_{\alpha}$. Let $a, b \in S$, and denote the D-classes containing $a$ and $b$ by $D_{a}$ and $D_{b}$ respectively. Then, by Lemma 1.1, $D_{a} \subseteq S_{\alpha}$ and $D_{b} \subseteq S_{\beta}$ for some $\alpha, \beta$ in $\Omega$. Hence $a b \in D_{a} D_{b} \subseteq S_{\alpha} S_{\beta} \subseteq S_{\alpha \beta}$ and $b a \in D_{b} D_{a}$ $\subseteq S_{\beta} S_{\alpha} \subseteq S_{\alpha \beta}$. Since $S_{\alpha \beta}$ is bisimple, we conclude that $a b D b a$. Thus (A) implies (B). (B) implies (C) by Lemma 1.2. Also (C) implies (D) trivially. We shall now show that (D) implies (C). Suppose $a \triangleright b$ and $c \in S$. Let $a^{\prime}, b^{\prime}, c^{\prime}$ be one of the inverses of $a, b, c$ respectively. Since $a^{\prime} a \mathfrak{L} a$ and $\mathfrak{L}$ is a right congruence, we have $a^{\prime} a \cdot c c^{\prime} \& a \cdot c c^{\prime}$. Also, since $c c^{\prime} R c$ and since $R$ is a left congruence, we have $a \cdot c c^{\prime} R a c$. Thus $a^{\prime} a c c^{\prime} D a c$ and likewise $b^{\prime} b c c^{\prime} D b c$. Since $a^{\prime} a D b^{\prime} b$, it follows from (D) that $a^{\prime} a c c^{\prime} D b^{\prime} b c c^{\prime}$ and hence $a c D b c$. In a similar manner, $c a D c b$ and thus $D$ is a congruence.

To show that (C) implies (E), suppose $a D b$ so that $a D b b^{\prime}$ where $b^{\prime}$ is an inverse of $b$. Since $D$ is a congruence, this implies that $a b D b b^{\prime} b$ or $a b D b$. Thus each D-class of $S$ is a subsemigroup of $S$ and moreover, since $S$ is regular, it is a bisimple semigroup [2, p. 61, Example 6]. Denoting the D-classes of $S$ by $S_{\alpha}, \alpha \in \Omega$, we have $S$ $=\bigcup\left\{S_{\alpha} \mid \alpha \in \Omega\right\}$. Further, since $D$ is a congruence, $S_{\alpha} S_{\beta}$ is contained in a semigroup $S_{\gamma}$ for some $\gamma \in \Omega$. If we define $\alpha \beta=\gamma$, then, noting that $S_{\alpha} S_{\alpha} \subseteq S_{\alpha}, \Omega$ becomes a band and thus $S$ is a band of bisimple semigroups.

Finally, in order to show that (E) implies (A), we use the following result by Clifford [1]. "Let $\mathcal{C}$ be a class of semigroups. If a semigroup $S$ is a band of semigroups of type $\mathcal{e}$, then $S$ is a semilattice of semigroups each of which is a rectangular band of semigroups of type e." Now assuming that $S$ is a band of bisimple semigroups, $S$ is a semilattice $\Omega$ of semigroups $S_{\alpha}(\alpha \in \Omega)$, where each $S_{\alpha}$ is a rectangular band of bisimple semigroups. If $a_{\alpha} \in S_{\alpha} \subseteq S$, since $S$ is regular, there exists $a_{\alpha}^{\prime} \in S$ such that $a_{\alpha} a_{\alpha}^{\prime} a_{\alpha}=a_{\alpha}$ and further $a_{\alpha} D a_{\alpha}^{\prime}$. However, by Lemma 1.1 each $S_{\alpha}$ is a union of D-classes of $S$ and hence $a_{\alpha}^{\prime} \in S_{\alpha}$. Thus $S_{\alpha}$ is regular, and in particular, quasiregular. Consequently, by Theorem 1.4, $S_{\alpha}$ is bisimple, and we have (A). This completes the theorem.

2. Examples. In this section we consider some examples to show that Theorem 1.5 is not true in general for semigroups which are not regular. First we need a lemma.

Lemma 2.1. Let $S$ be a Baer-Levi semigroup [3, p. 82] and $T$ be any semigroup. Let $A=T \times S$ be the direct product of $T$ and $S$. Then $(t, s)$ $\mathbb{D}\left(t^{\prime}, s^{\prime}\right)$ if and only if $t R t^{\prime}$, in $T$, and consequently the $D$-classes of $A$ are just the sets $R \times S$ where $R$ is an $R$-class of $T$. 
Proof. The proof of the "only if" part follows if we show that the $\mathfrak{L}$-classes of $A$ contain single elements. Now suppose $(t, s) \mathfrak{L}\left(t^{\prime}, s^{\prime}\right)$. Then either

(i) $(t, s)=\left(t^{\prime}, s^{\prime}\right)$, or

(ii) there exists $x, y$ in $T$ and $a, b$ in $S$ such that $(x, a)(t, s)=\left(t^{\prime}, s^{\prime}\right)$ and $(y, b)\left(t^{\prime}, s^{\prime}\right)=(t, s)$.

If (ii) holds, we have $a s=s^{\prime}$ and $b s^{\prime}=s$. Thus, $(b a) s=b(a s)=b s^{\prime}=s$ which contradicts [3, Lemma 8.3]. Hence (ii) cannot hold, and we have established the assertion.

The "if" part is clear, since $S$ is right simple and any two elements of $S$ are R-related.

ExAmple 1. Let $E_{1}=\left\{e_{1}, e_{2}\right\}$ be a right zero semigroup of order 2, $E_{2}=\left\{e_{3}, e_{4}, e_{5}\right\}$ be a left zero semigroup of order 3, and let $E=E_{1} \cup E_{2}$ be a disjoint union where

$$
\begin{aligned}
\quad e_{i} e_{j}=e_{j} e_{i}=e_{j} \quad \text { for } i=1,2, j=3,4, \\
e_{1} e_{5}=e_{3}, \quad e_{5} e_{1}=e_{5}, \quad e_{2} e_{5}=e_{4}, \quad e_{5} e_{2}=e_{5} .
\end{aligned}
$$

The fact that $E$ is a semigroup can be easily verified. Moreover, $e_{1} R e_{2}$ and $e_{3} \& e_{4} \& e_{5}$. Set $T=E \times S$, the direct product of $E$ and $S$, where $S$ is the Baer-Levi semigroup. Using Lemma 2.1, the D-classes of $T$ are given by

$$
D_{1}=\left\{e_{1}, e_{2}\right\} \times S, \quad D_{i}=\left\{e_{i+1}\right\} \times S(i=2,3,4) .
$$

Clearly, $T$ is a band of the bisimple semigroups $S_{i}=\left\{e_{i}\right\} \times S, i=1$, $2,3,4,5$. However, if $s \in S$, then $\left(e_{1}, s\right) D\left(e_{2}, s\right)$. But $\left(e_{1}, s\right)\left(e_{5}, s\right)$ $=\left(e_{3}, s^{2}\right) \in D_{2}$ and $\left(e_{2}, s\right)\left(e_{5}, s\right)=\left(e_{4}, s^{2}\right) \in D_{3}$. Thus $D$ is not a congruence. Moreover, $T$ is not a semilattice of bisimple semigroups, for otherwise, Lemma 1.3 would imply that $D$ is a congruence. Further $T$ does not satisfy the condition (B) of Theorem 1.5, since in that case Lemma 1.2 would again imply that $D$ is a congruence.

ExAmple 2. Let $S=\{0, a, 1\}$ where $a^{2}=0$, with 0 as the zero element and 1 as the identity element. Then $D$ is a congruence, being the identity relation. However, $S$ is neither a band of bisimple semigroups nor a semilattice of bisimple semigroups.

EXAMPLE 3. Let $E=\left\{e_{1}, e_{2}\right\}$ be a left zero semigroup and let $S$ be the Baer-Levi semigroup. Set $T=E \times S$. Then $T$ is a semigroup and by Lemma 2.1, the D-classes of $T$ are:

$$
D_{1}=\left\{e_{1}\right\} \times S \text { and } D_{2}=\left\{e_{2}\right\} \times S .
$$

Moreover, $D_{1}$ and $D_{2}$ are bisimple semigroups such that $D_{1} D_{2} \subseteq D_{1}$ and $D_{2} D_{1} \subseteq D_{2}$. Thus $D$ is a congruence on $T$ and $T$ is a band of bi- 
simple semigroups. However, if $a=\left(e_{1}, s\right)$ and $b=\left(e_{2}, s\right)$ for $s \in S$, then $a b \in D_{1}$ and $b a \in D_{2}$. Hence, $a b$ and $b a$ are not D-related. This will further imply that $T$ is not a semilattice of bisimple semigroups.

REMARK. If, in Example 3 above, we write $S_{11}=D_{1}, S_{21}=D_{2}$, we find that $S$ is a rectangular band of the bisimple semigroups $S_{11}$ and $S_{21}$. But $S$ is not bisimple. This shows that Theorem 1.4 is not true in general for semigroups which are not quasiregular.

This paper is part of a Ph.D. thesis submitted to the University of Wisconsin-Milwaukee. I would like to thank Dr. R. L. Gantos for his valuable advice and encouragement.

\section{REFERENCES}

1. A. H. Clifford, Bands of semigroups, Proc. Amer. Math. Soc. 5 (1954), 499-504. MR 15, 930.

2. A. H. Clifford and G. B. Preston, The algebraic theory of semigroups. vol. I, Math. Surveys, no. 7, Amer. Math. Soc., Providence, R. I., 1961. MR 24 \#A2627.

3. - The algebraic theory of semigroups. vol. II, Math Surveys, no. 7, Amer. Math Soc., Providence, R. I., 1967. MR 36 \#1558.

4. Josette Calois, Demi-groups quasi-inversifs, Academie des Sciences, Seance du 17, April, 1961, pp. 2357-2359.

5. W. D. Munn, Regular w-semigroups, Glasgow Math. J. 9 (1968), 46-66. MR 37 \#3316.

The University of Wisconsin-Green Bay, Green Bay, Wisconsin 54301 